\title{
Ignames, enfants des hommes. Horticulture et reconduction du social à Wallis (Polynésie occidentale)
}

\section{Sophie Chave-Dartoen}

\section{CpenEdition}

\section{Journals}

Édition électronique

URL : http://journals.openedition.org/jso/6057

DOI : $10.4000 /$ jso. 6057

ISSN : 1760-7256

Éditeur

Société des océanistes

\section{Édition imprimée}

Date de publication : 15 décembre 2010

Pagination : 145-160

ISBN : 978-2-85430-027-7

ISSN : 0300-953x

Référence électronique

Sophie Chave-Dartoen, « Ignames, enfants des hommes. Horticulture et reconduction du social à Wallis (Polynésie occidentale) », Journal de la Société des Océanistes [En ligne], 130-131 | 2010, mis en ligne le 15 décembre 2013, consulté le 09 juin 2020. URL : http://journals.openedition.org/jso/6057 ; DOI : https://doi.org/10.4000/jso.6057 


\section{Ignames, enfants des hommes. Horticulture et reconduction du social à Wallis (Polynésie occidentale) ${ }^{1}$}

par

Sophie CHAVE-DARTOEN*

\section{RÉSUMÉ}

Cet article, qui présente le travail horticole et une partie des responsabilités rituelles des hommes à Wallis (Polynésie occidentale), porte sur les ignames (mise en culture, classifications indigènes, système rituel et circuits d'échange) et montre que ces tubercules participent d'un vaste système de relations qui définissent tout être en référence à la société wallisienne dont le "roi » scelle l'ensemble de l'organisation. Comparables à des enfants masculins que les hommes obtiennent de leurs jardins, les ignames concourent, en circulant dans les échanges, à la constitution différenciée des vivants, au renouvellement du monde et à la pérennisation de la société.

Mots-CLÉS: anthropologie sociale, Wallis ('Uvea), Polynésie occidentale, igname, techniques horticoles, système rituel, composantes de la personne

\begin{abstract}
This paper describes a part of the men's responsibilities, both agricultural and ritual, in Wallis Island, Western Polynesia. Yams are the main focus of this study including agricultural techniques, indigenous classifications, ritual system and exchange pathways. It shows that yams are involved in a large system of relationships that defines everything in relation with the society itself. Comparable to sons that men would get from their gardens, these tuber partake, by circulating in exchanges, in the differentiated constitution of living people, the world's renewing and the society's everlastingness.
\end{abstract}

KeYwORDs: social anthropology, Wallis ('Uvea), Western Polynesia, yams, agricultural techniques, ritual system, constitution of the person
« La vision symbolique yafar du monde vivant renvoie la production à la reproduction : produire un jardin, c'est mettre en place un chaînon unique d'une chaîne reproductive, ininterrompue, qui assure la permanence de la vie. Défrichements, brûlis, bouturages, désherbages en forment le niveau matériel et techni- que ; démarcations, attributions, héritages, distributions en sont les formes de contrôle social ; représentations cosmologiques et rituels en fournissent le cadre imaginaire et en garantissent l'efficacité. » (Juillerat, $1986: 171-72)$

1. Cet article reprend, de façon très remaniée, un développement de ma thèse de doctorat (Chave-Dartoen, 2000). Il résulte d'une recherche de terrain à Wallis, seize mois entre 1992 à 1994 et trois mois en 2007. Je remercie les personnes et les institutions ayant permis ce terrain (Fondation de la Maison des sciences de l'homme, GDR CNRs 748 et allocation « Jeune chercheur» de l'École des hautes études en sciences sociales pour le premier séjour, Équipe d'accueil 2963 et BQR de l'Université Bordeaux 2 pour le second). Je trouve ici l'occasion de remercier les villageois de 'Utufua et de nourrir une pensée toute spéciale pour Pelesese Tokotu'u, Aliki Liufau et Setino Siuli, tous trois décédés depuis cette enquête. Je leur dois les connaissances mobilisées ici. Merci également à Paino Tau'ota pour son assistance sur place et à Claudine Friedberg pour sa lecture attentive et ses judicieuses remarques.

* EA2963 atotem, Université Bordeaux 2 Victor Segalen, sophie.chave-dartoen@u-bordeaux2.fr. 
Dans Les enfants du sang, la remarquable monographie que Bernard Juillerat a consacrée à l'organisation et à l'imaginaire de la société yafar (Papouasie Nouvelle-Guinée), le chapitre relatif à l'horticulture laisse peu explicite l'articulation entre la culture des jardins et les représentations qui s'y rattachent. Ce travail présente, en effet, le vivant comme le substrat matériel d'une élaboration imaginaire et symbolique qui informe le social tout en s'en distinguant :

« Après s'être protégé - préalablement à l'acte de transgression que représente cette violence nécessaire sur la nature - de la culpabilité et des stigmates d'un retour trop abrupt au maternel, l'homme peut alors plus à son aise manipuler les principes et les substances reproductives et se donner ainsi l'illusion et le pouvoir de contrôler la fertilité de la nature et la production des essarts. » (1986: 174)

La formulation est ambiguë, «l'illusion et le pouvoir » étant ici conjoints là où « l'illusion du pouvoir » eut été une formule plus respectueuse de la dissociation de domaines - biologique, social et psychique - que l'auteur pose comme des réalités différentes, mais dont les analyses ne définissent pas, dans ce contexte précis, la portée et les limites. Afin d'éclairer ce point, il eût sans doute été intéressant qu'il précisât, pour ce qui concerne l'horticulture, la nature exacte des représentations cosmologiques et des rituels en mesure tout à la fois de fournir le « cadre imaginaire » et de garantir l'« efficacité » de la reproduction biologique et sociale ( $c f$. citation en exergue). Ce chapitre laisse donc ouverte la question de l'articulation du symbolique et du social, le premier étant tantôt dans, tantôt hors du second ${ }^{2}$.

En guise d'hommage, je reprendrai ici le problème des représentations et de leur efficacité à partir de ce que Bernard Juillerat appelle la " production des jardins », mais en partant d'un autre terrain. Mes recherches (Chave-Dartoen, 2000) sur l'organisation sociale et le système rituel de la société de l'île Wallis - rites de passage et échanges cérémoniels - montrent que l'importance rituelle des plantes cultivées, des techniques et des pratiques qui leur sont associées ne peut être pleinement saisie à travers une opposition entre matériel et symbolique. Les Wallisiens, par exemple, font une nette distinction entre éléments humains et non humains du cosmos, mais les réalités matérielles sur lesquelles ces catégories reposent échappent au cadre de notre propre matérialité : la présence des morts est tangible à travers le danger effectif qu'ils représentent pour les vivants (maladie, infortune, décès) et pour les jardins (infestations, infertilité, sécheresse). De façon plus générale, une interprétation en termes de dichotomie êtres/choses ou sujet/objet serait abusive : animaux, pierres et plantes sont intégrés au même titre que les humains au monde socialisé, mais à différents niveaux. Dans de tels cas, l'analyse ne peut se fonder que sur les catégories vernaculaires et en creuser les principes internes sans préjuger de domaines proprement biologiques, sociaux ou psychologiques.

Dans l'exemple polynésien que je développerai ici, horticulture et circulation des récoltes ne sont pas dissociables. Je ne présenterai pas, faute de place dans cet article, l'ensemble des représentations et des pratiques liées aux plantes cultivées. De même, laissant de côté l'analyse de la circulation des porcs et des biens féminins, je n'aborderai pas l'ensemble du système rituel où hommes et femmes partagent des charges et des responsabilités différentes (Chave-Dartoen, 2000). Je montrerai en revanche qu'à Wallis, la chair des plantes cultivées, leur matière constitutive, est complétée, tout comme celle des humains, par d'autres principes relationnels et que tous participent d'un même système de circulation. À travers cette approche, pratiques et pensée wallisiennes apparaissent infiniment plus complexes que saisies au prisme de nos propres catégories. Pour une telle société, se comprenant elle-même comme une totalité cohérente, l'étude des pratiques horticoles doit nécessairement s'ouvrir aux systèmes d'échange et aux cycles rituels.

\section{Présentation de la société de l'île Wallis}

Wallis ('Uvea en langue vernaculaire) est un archipel dont l'île principale (environ $95 \mathrm{~km}^{2}$ ) est encerclée d'un lagon bordé par une vingtaine d'îlots épisodiquement habités. Elle est située par $13^{\circ} 20^{\prime}$ de latitude sud et $176^{\circ} 10^{\prime}$ de longitude ouest, au centre de l'aire culturelle appelée Polynésie occidentale (Tonga et les Fidji de l'est, Futuna, Tokelau et Samoa). Son climat subtropical est soumis à l'alizé austral de mai à octobre et aux cyclones, parfois dévastateurs, de novembre à avril.

Territoire d'outre-mer depuis 1961, Wallis et la proche Futuna forment une collectivité

2. «Partis du juridique et parvenus jusqu'au seuil des structures symboliques (et psychiques), nous voilà hors du social » (Juillerat, 1986 : 174). Dans les articles qui suivront la monographie (1986) et dans son dernier ouvrage (2001), les analyses de Bernard Juillerat soumettent clairement certains phénomènes sociaux, tel le système rituel yafar, à des processus psychologiques accessibles au moyen d'une lecture psychanalytique. 
d'outre-mer depuis 2003. Jusqu'à ces dates récentes, le rôle colonial de la France dans ce lointain protectorat fut essentiellement indirect et largement fondé sur la présence de la mission mariste qui, implantée sur place en 1837, évangélisa et convertit la population en quelques années. Sous son influence, l'organisation sociale se modifia : le mariage entre membres d'ordres sociaux autrefois endogames fut favorisé ; des communautés villageoises rassemblèrent sur la côte la population qui vivait plus dispersée; la chefferie, qui regroupait autour d'un "chef suprême » hau les hommes forts du moment, prit une forme fixe autour du hau, terme désormais traduit par « roi». Depuis lors, la chefferie se compose d'un conseil de six membres ('aliki fau) et de l'ensemble des chefs de village (pule kolo). Le « roi » (hau) établit une relation privilégiée avec Dieu dont il fait bénéficier en retour l'ensemble de ses dépendants. Clef de voûte de l'organisation sociale, il dirige les rituels et préside l'ensemble des cérémonies qui ont intégré, en se perpétuant, valeurs et représentations chrétiennes. La population actuelle (environ 10000 habitants) est répartie dans une vingtaine de villages ${ }^{3}$.

La végétation prend à Wallis trois aspects différents. Le premier est la forêt tropicale dense (va'o matu'a), partiellement conservée au sudouest, dont on dit qu'elle préserve l'humidité et la fertilité du pays (Guiot, 1998). Le roi, qui en est responsable, y autorise occasionnellement des prélèvements de bois d'œuvre ou une mise partielle en cultures si la nécessité est pressante. La deuxième sorte de végétation, au centre et au nord de l'île, forme un maquis (toafa) résultant d'une exploitation trop intense de sols fragiles partiellement latérisés. La troisième, qui ceinture l'île, est une couverture de jardins vivriers et de friches dominés par les cocotiers dont de grandes plantations s'étendent au sud de l'île.

\section{La culture des jardins ${ }^{4}$}

À Wallis, la culture des jardins, considérée comme pénible, ne convient pas aux femmes qui ont «les bras faibles» (nima vaivai). Ces dernières s'aventurent d'ailleurs rarement dans les jardins, lieux isolés communément associés aux relations intimes des couples mariés ou clan- destins. En marge de la zone d'habitation côtière, les jardins se trouvent en outre sur les terres intérieures où rôdent les morts à l'action néfaste desquels les femmes sont potentiellement plus sensibles. Jardins et horticulture relèvent ainsi de l'entière responsabilité des hommes et d'un travail génésique qui associe récoltes végétales et procréation humaine.

Les cultures vivrières demandent le défrichement et l'entretien régulier de grandes surfaces de terrain. Cependant, les conditions de ce travail changent profondément depuis quelques années sous l'effet combiné du manque de temps des salariés, de la rareté des terres disponibles et des facilités qu'apportent sur l'île techniques et technologie occidentales (véhicules motorisés, tronçonneuses, engrais chimiques...). À cela s'ajoute la transformation des besoins cérémoniels et alimentaires : les vivres importés obtenus dans le commerce intègrent de façon croissante échanges et repas. Alors que dans chaque « maisonnée " ('api, au sens d' " unité familiale de base »), les hommes défrichaient au moins un jardin chaque année il y a encore quelques décennies, ils ne défrichent plus en moyenne qu'une parcelle tous les deux ou trois ans ${ }^{5}$.

Chaque parcelle est prise sur les « terres familiales » (kele fakafamili), c'est-à-dire sur les terres dont les droits d'usage, hérités des ancêtres paternels et maternels, sont indivis dans l'ensemble des descendances. Une fois le lopin choisi et le consentement des co-ayants droit acquis, la végétation est coupée et brûlée. Les arbres, que l'on a fait mourir en entretenant le feu à leur pied, sont laissés en place. Les semences sont préférentiellement plantées à leur base, contre les troncs ou près des pierres affleurant à la surface du sol dont on considère qu'elles fertilisent la terre en lui conservant son humidité. Les arbres morts constituent un support pour les tiges des ignames plantées autour d'eux. Le parti ainsi tiré des opportunités du terrain donne aux plantations une apparence désordonnée caractéristique.

Les végétaux sont traditionnellement reproduits par division et reproduction des clones (tubercules) ou par bouturage (maniocs et plantes ornementales). La chaleur humide de la région convient aux plantes subtropicales qui poussent sans soin particulier 6 sinon le

3. NDLR. - Pour plus d'informations sur Wallis-et-Futuna, voir le JSo 122-123 Spécial Wallis-et-Futuna (H. Guiot et I. Leblic éds) paru en 2006 et intégralement en ligne sur jso.revues.org.

4. Les techniques agricoles et le calendrier agraire de Wallis ont été présentés par Patrick Vinton Kirch (1978).

5. Les pratiques varient selon les ressources dont disposent les maisonnées et leur disponibilité en force de travail. Dans certains cas, l'horticulture est un moyen de gagner un peu d'argent via la commercialisation des fruits du travail, mais une telle activité est peu considérée et marginale (Grijp, 2002).

6. Pour un inventaire plus détaillé des plantes cultivées, on peut se reporter à l'article de Jacques Barrau (1963). 


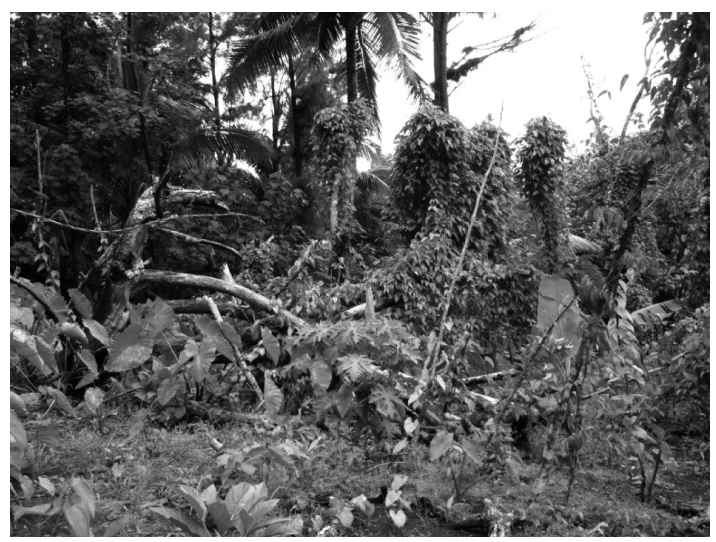

Рното 1. - Les jardins wallisiens ont généralement un aspect assez désordonné, des plantes de différentes espèces (ici ignames, taros, papayers, bananiers, cocotiers...) s'y trouvant à différents stades de développement (cliché de l'auteur, janvier 2008)

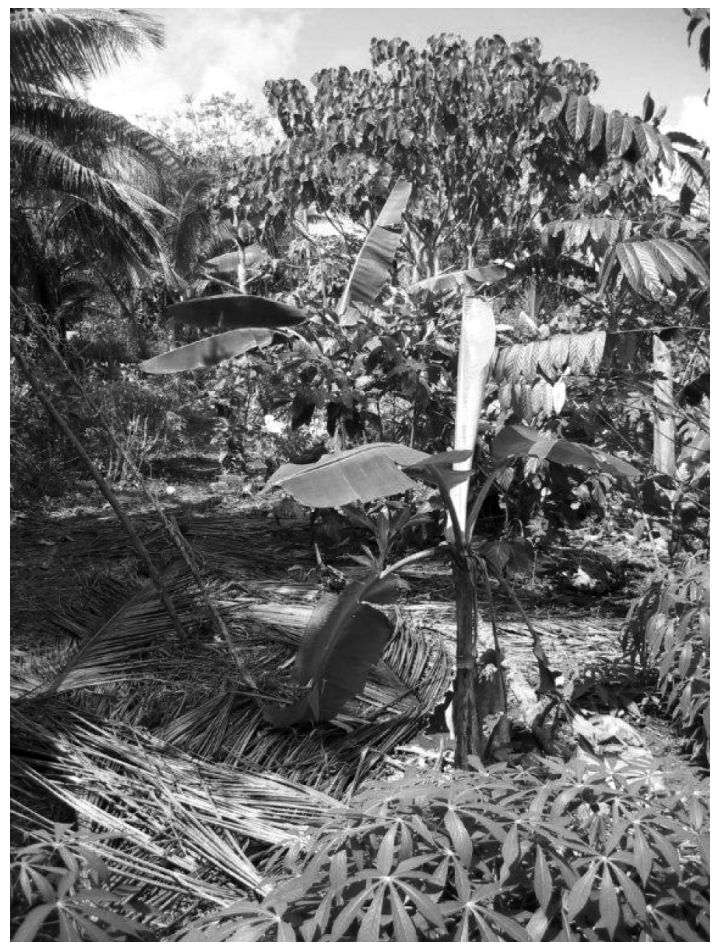

Рното 2. - Des plants de manioc sont plantés après les premières récoltes d'ignames et de taros (cliché de l'auteur, juillet 2005)

nettoyage régulier du terrain autour d'elles. Selon P. Tokotu'u, ancien cultivateur résidant à Utufua, une "récolte» $(t a$ 'u, également «saison») doit être plantée durant les trois jours de pleine lune et les trois jours de nouvelle lune, ce qui assure les meilleurs résultats. $\mathrm{Ce}$ calendrier lunaire n'est désormais plus respecté et, dans l'ensemble, les plantations sont faites « n'importe quand » (ta'uvale), suivant la disponibilité du cultivateur, ses ressources en semences et les besoins programmés de la maisonnée. Des récoltes supplémentaires sont planifiées lorsque des cérémonies sont prévues à long terme.

De nos jours donc, les techniques et le rythme de mise en terre varient selon les plantes cultivées et les récoltes attendues. Les Aracées (principalement taros Colocasia esculenta ${ }^{7}$ et kape Alocasia macrorrhiza, Shott.), par exemple, sont replantées au fur et à mesure qu'elles sont récoltées de telle sorte qu'un jardin comprend généralement des tubercules à différents stades de croissance. Le manioc, très secondaire dans l'alimentation humaine, assure une bonne part de celle des porcs, nombreux et voraces. Les bananiers $\left(f u^{\prime} u \text { fusi }\right)^{8}$ ne font l'objet d'aucun soin particulier. Ils fructifient tout au long de l'année (des variations existent selon les espèces) avec une période d'abondance en juillet-août. Les arbres à pain (fu'u mei, Artocarpus altilis [Parkinson] Fosberg) ${ }^{9}$ fructifient de décembre à février, puis de nouveau en juin-juillet. Les ignames sont les seules plantations saisonnières organisées en trois récoltes annuelles. Particulièrement exigeantes en travail, elles sont indispensables aux prestations masculines lors des nombreuses cérémonies qui rythment la vie locale.

\section{Les ignames, prestations masculines par excel- lence}

Bien qu'elles soient rarement présentées seules, les ignames crues constituent le volet masculin des prestations cérémonielles par excellence. Individuelles ou collectives, ces prestations sont présentées en de multiples occasions (rites de passage, célébrations liturgiques, propitiations), généralement accompagnées de leur volet féminin normalement constitué de nattes et de tissu d'écorce battue (Chave-Dartoen, 2000).

Les ignames suivent deux circuits d'échange différents. Le premier, cérémoniel, correspond aux obligations que tout homme doit à ses « anciens » et à ceux de la communauté : des prestations ('umu, «[contenu de] four») sous forme de piles (ou de paniers) d'ignames crues surmontées d'un porc cuit au four (ou passé au feu) sont présentées pour les grandes cérémonies

7. Les variétés recensées sont : magasiva ; talo fisi « taro de Fidji»; talo’uli « taro noir »; manu'a « taro de Manu'a »; talo vale « taro fou »; talo sika « taro pointe de lancette », probablement tous Colocasia esculenta ; maga'uli, qui a disparu (pour un inventaire plus complet voir Chave-Dartoen, $2000: 110$ note 70 et Barrau, $1963: 164$ ).

8. Il existe plusieurs variétés de Musacées caractérisées par la qualité de leurs fruits (selon Barrau [1963: 165], toutes sont des Eumusa).

9. Il existe plusieurs variétés de ces Moracées. Elles se distinguent par la forme des feuilles et la qualité des fruits. 
publiques (principalement les fêtes religieuses et patronales, les investitures et l'accueil d'hôtes de marque). Les hommes en offrent aussi lors des cérémonies du cycle de vie, des rites de propitiation et des déplacements de leurs relations kaiga. Le premier cercle des relations kaiga est celui des proches parents. L'étendue de ces relations est cependant beaucoup plus vaste puisque s'y combinent, outre l'ascendance commune et le mariage, des critères de co-résidence ou d'affinités personnelles. Théoriquement innombrables, ces relations sont socialement validées par la solidarité dans les obligations cérémonielles. Seule cette dernière leur confère existence et actualité.

Le second circuit est beaucoup moins formel. Il suit le même réseau de relations (kaiga), mais cette fois, afin d'obtenir, tous les trois ans, de nouvelles semences de différentes variétés pour les récoltes à venir. Cette circulation est capitale : à défaut, les récoltes de la quatrième année sont infructueuses; la semence germe, puis les ignames dépérissent :

«Tu t'en occupes [tu plantes la semence] et elle disparaît $^{10}$ ! (Pelesese Tokotu'u, extrait d'entretien)

Je vais revenir sur ces deux circuits de circulation après une rapide présentation des tubercules, des sortes de semences que l'on prépare et du travail horticole que cela implique.

Dans le langage courant, toutes les ignames
(Dioscorea) relèvent de la catégorie générale des 'ufi' ${ }^{11}$. Il en existe, à Wallis, de nombreux cultivars locaux (fa'ahiga 'ufi) ${ }^{12}$ qui s'organisent en sousensembles de taille et de contenu variables, l'étendue et la complexité des savoirs naturalistes dépendant largement des compétences des informateurs. Sur pied, les ignames sont identifiées à l'aspect des feuilles et de la tige. Une fois arrachées, l'identification porte sur la morphologie du tubercule : taille, forme et qualité de la peau auxquelles s'ajoutent couleur, parfum et texture de la chair. Le jeu de ces critères permet, selon les contextes, des comparaisons et des classifications empiriques du type " petites ignames rouges », « ignames parfumées » ou « ignames à développement horizontal »... Lors d'un entretien consacré à la question, $\mathrm{P}$. Tokotu' $\mathrm{u}^{13}$ indiqua par exemple que les ignames vegi ${ }^{14}$, sikau et lausi étaient «mélangées ensemble » (natu fakatahi) dès la récolte selon des critères croisant taille, valeur gustative et valeur cérémonielle. Il revint ensuite sur la question précisant que si, toutes les ignames de belle taille et de bel aspect peuvent convenir pour les prestations, les ignames considérées comme originaires de Wallis sont toutefois préférables. Dans cette série, s'accolent aux lausi les grandes ignames poa et laumahi. A contrario ne figurent pas dans les prestations le sous-ensemble 'ufilei'15 (Dioscorea esculenta [Lour] Burk) et toutes les ignames sauvages que l'on déterre dans les friches ${ }^{16}$ pour la consommation immédiate ou pour être

10. «E ke taupau ai pe, e alu o puli! » (Pelesese Tokotu'u, extrait d'entretien).

11. Jacques Barrau (1963:165) ne parle pas de catégorie englobante ('ufi) et distingue : 1-ignames sauvages : hoi (Dioscorea bulbifera) et lena (Dioscorea pentaphylla L.) ; 2- ignames cultivées : 'ufi, (Dioscorea alata L. dont il recense vingt-deux variétés) et 3- 'ufi lei (Dioscorea esculenta Burk). Une espèce (Dioscorea nummularia Lamk.) recoupe les deux premières catégories : sa variété cultivée est appelée 'ufi fiti, ses deux variétés sauvages sont appelées palai et tu'akuku.

12. Dans son dictionnaire, Karl Rench (1984), répertorie à l'entrée « 'ufi » quarante-trois sortes d'ignames dont vakasoa, voli, vegi, poa, niumea, heketala, tu, salomone, fisi, kula, la'usi, lena, palai, kahokaho, palepale, tu'akuku, laumahi, kaumaile... Les ignames de cet inventaire sont données comme Dioscorea alata.

13. Nous passâmes trente-trois «sortes d'ignames » en revue avec Pelesese Tokotu'u lors d'un long entretien mené avec P. Tau'ota. L'essentiel des informations de P. Tokotu'u citées ici me furent données à cette occasion. 'Ufi Vegi peut être traduit par « igname de Vegi », 'ufi lausi « ignames feuilles-de-cordyline », 'ufilei (« igname-dent-de-cachalot»), un travail de sémantique reste à faire pour éclaircir les autres noms.

14. Les 'ufi vegi sont remarquables et qui très recherchées à Wallis. Elles ont été apportées au début du $\mathrm{Xx}^{\mathrm{e}}$ siècle par un Chinois nommé Vegi (information P. Tokotu'u). Elles sont présentées, dans les prestations cérémonielles, avec les variétés considérées comme indigènes. Très résistantes aux intempéries, elles « ne disparaissent jamais ». Le fait est notable car, selon $P$. Tokotu'u, les ignames importées, pourtant très recherchées, s'adaptent mal et toutes finissent par s'éteindre. Sont également d'importation récente, les hoi (Barrau donne les hoi pour une espèce sauvage dont la cueillette est occasionnelle) appelées 'ufi mālie à Nouméa d'où elles sont originaires, les 'ufi niumea («ignames de Nouméa »), les 'ufi manioka (igname-manioc ou 'ufi kaletonia "ignames de Calédonie »). Les 'ufi kahokaho qui proviennent de Tonga (où des ignames du même nom sont considérées comme supérieures à toutes les autres), et les 'ufi vakasoa (vakasoa matapoko, vakasoa loloa) originaires de Futuna sont probablement d'importation plus ancienne.

15. L'espèce comprend les kautala, les lavilavi, les 'ufilei vai et les lavilavi kula. Jacques Barrau (1963 : 165) mentionne aussi les lotuma («Rotuma »). De texture très tendre, les 'ufilei sont gardées pour la consommation courante (on ne les présente que si elles atteignent une taille exceptionnelle).

16. Autrefois, les ignames sauvages constituaient une ressource importante en temps de disette, avec les tubercules appelées pulaka (Cyrtosperma chamissonis) et une plante appelée mahoa'a (arrowroot, Tacca leontopetalö̈des [L.]). La riche végétation du district sud devait assurer des ressources en noix de coco et en végétaux non cultivés dont ne bénéficiaient pas les communautés installées au nord. Les variations pédologiques et botaniques de l'île peuvent expliquer qu'au sud on ne préparait pas (ou très peu) de puits à mahi, fosses tapissées de feuilles où étaient conservés, pour assurer les soudures, des fruits de l'arbre à pain ou certaines bananes fermentés. 
replantées dans le jardin : tu'a kuku, palai (toutes deux Dioscorea nummularia Lamk. ${ }^{17}$ ), tuu pakata (originaires de Futuna), kaukau si'i et lena (Dioscorea pentaphylla L.).

Les ignames idéales, et particulièrement celles nécessaires aux prestations cérémonielles, doivent ainsi remplir deux critères : l'un, morphologique, conjugue taille du tubercule et qualité de la peau ; l'autre, plus proprement sociologique, relègue les cultivars considérés comme sauvages ou importés. Seules conviennent parfaitement aux prestations les ignames du " pays » ('Uvea) combinant caractère local et la culture en jardin. Ces considérations prennent tout leur relief si on les rapporte à d'autres aspects de la culture et de la circulation des tubercules.

\section{La culture des ignames, travail des hommes}

Ce qui caractérise les ignames et leur confère une valeur particulière, c'est l'expertise et la somme d'efforts que leur reproduction et leur culture requièrent. Leur technique de reproduction est végétative et, dans toute la région, les cultivateurs, conscients du fait que les plantes descendant d'une même souche présentent les mêmes caractéristiques, en usent pour opérer une fine sélection des cultivars (Lebot, 2002). Pour chaque variété, quelques tubercules de bonne taille et de bel aspect (finesse et clarté de la peau) sont mis de côté de façon fournir les semences de la récolte correspondante pour l'année à venir. Les tubercules sont alors jamais coupés mais «divisés» (tofi), le tranchant du couteau, tout juste enfoncé dans la chair, faisant levier pour la rompre.

Il existe deux sortes de « semences » (pulapula $^{18}$ ) dont la distinction est capitale pour comprendre le cycle agricole et l'organisation des circulations cérémonielles. Le sommet des tubercules, appelé en français comme en wallisien "tête de l'igname" ('ulu'i'ufi), est mis de côté dans des paniers de sable où il germe rapidement avant d'être replanté. Du reste de chaque tuber- cule ( « corps de l'igname », sino'i'ufi) on tire des semences appelées "semenceaux» en français, fakafale en wallisien ( faka « qui ressortent de, de l'ordre de »; fale " maison »). Plus nombreux, ces semenceaux sont aussi plus lents à germer (ils étaient autrefois placés sur des nattes de cocotier, dans la charpente de la maison) et sont plantés dans un second temps. Pour chaque récolte, les fruits des semenceaux (fakafale) sont donc plus tardifs que ceux des têtes ('ulu'i'ufi). Mentionnons que ces derniers connaissent une distinction secondaire avec les "vraies têtes d'ignames » ('ulu'i'ufi totonu) sur laquelle je reviendrai en présentant les différentes sortes de récoltes et de prestations.

La préparation d'une récolte consiste aussi à essarter les friches, ameublir la terre au bâton à fouir pour y planter les semences soigneusement préparées sous une faible épaisseur de terre ou, mieux encore, de sable fin. Cette séquence revient à un « travail vers le bas » (gaue'ifo), locution par ailleurs employée pour désigner l'action de l'homme lors d'un accouplement. Des tuteurs, dont les faisceaux supporteront les tiges régulièrement ligaturées, sont préparés et installés. Les tiges se développent en hauteur sur trois à cinq mètres et se couvrent de feuilles, formant de hautes silhouettes végétales dressées vers le ciel. Durant ce temps, les jardins sont nettoyés et protégés des porcs vagabonds. Après six ou sept mois de croissance, la plante dépérit. Vient alors le moment de la récolte. L'extraction est délicate car les tubercules ne doivent pas être abîmés ou cassés. On s'aide pour cette opération d'un pieu de bois ou d'une barre à mine et d'un sabre d'abattis. L'obtention de tubercules de grande taille dépend de différents facteurs : le choix de variétés appropriées (vegi, lausi, laumahi ou poa) ; la qualité et la préparation du sol, riche et profondément ameubli ; la taille des semences, dont on ne conserve que le germe le plus vigoureux avant la plantation ${ }^{19}$; la dextérité du cultivateur, lors de la récolte. La jachère de cinq à dix ans - souvent moins ${ }^{20}$ - et le brûlis sont les

17. Si les ignames palai et tu'a $k u k u$ ne figurent pas crues dans les prestations, elles y figurent sous forme de pâte d'igname cuite à l'étouffée avec du lait de coco $(l \bar{u})$ que la population du district de Mu'a prépare à l'occasion de la fête paroissiale de la Saint-Joseph, le $1^{\text {er }}$ mai. Ce jour-là, ces préparations, qui complètent des paniers de vivres cuits au four, sont distribuées aux membres de la chefferie et du clergé ainsi que chez les parents et les amis dispersés dans l'île.

18. Le terme pula signifie en wallisien « bouillir, bouillonner, pétiller, éblouir » (Rensch, 1984), le redoublement du lexème signifiant une atténuation. Si pulapula désigne une semence, une graine, sa signification revient plus particulièrement à l'apparence de légère effervescence qu'implique une germination.

19. Il existe une technique appelée tei'ulu. Elle vise à prélever un tubercule dans le jardin en replantant immédiatement la tête (P. Tokotu'u, extrait d'entretien).

20. La durée de la jachère dépend des terres disponibles pour la rotation. Une jachère ancienne couverte d'arbres est appelée « brousse boisée » vao 'akau'i, mais il existe des étapes intermédiaires qui dépendent en partie de la nature du sol. Citons le $m o o k u$, une « brousse blanche» (e kau hina), herbeuse et le saulagi qui est « rouge » (e kau kula). 
seules techniques assurant la régénération du $\mathrm{sol}^{21}$.

L'étendue et le nombre des plantations d'ignames (gaue'ufi), qui autrefois couvraient une grande partie des jardins, ont considérablement diminué depuis l'intensification du travail salarié et le désintérêt des jeunes générations pour les travaux horticoles. S'il est important qu'un homme fournisse des prestations appropriées en ignames et en porcs, un adage récurrent stipule que l'on n'offre jamais que ce que l'on peut donner, y compris pour le roi. La maîtrise de cette culture exigeante rehausse ainsi le statut des horticulteurs " grands travailleurs » (fa'a gaue) qui entretiennent de riches jardins et fournissent des prestations hors de la moyenne face à ceux qui, plantant au plus près des besoins, se reposent sur l'assistance de leurs relations kaiga ou complètent occasionnellement leurs prestations par des biens de substitution (sucre, riz, farine, numéraire...). Ainsi la qualité des prestations et l'honneur qui en découle varient selon les horticulteurs. Le fait que l'énumération cérémonielle des prestations use généralement d'une tournure discursive dépréciative ne présage en rien de l'évaluation réelle qui suit les discours sous forme de compliments ou de plaisanteries amusées. Il existe cependant une norme pour la « prestation en ignames convenant au roi » (fuahaumālie, plus littéralement "offrande vivrière digne du roi en ce qu'elle est non pourrie » selon le père S-L. Ikauno) : elle se compose des premières ignames issues de la «toute première récolte » (ta'u mu'amu'a). Donc, pour les offrandes au roi, toutes les prestations et toutes les ignames ne se valent pas.

\section{Les « récoltes » $(t a ’ u)$ d'ignames}

La culture des ignames était, pour les anciens du village, l'activité principale des hommes. Durant leur jeunesse, chacun entretenait conjointement une plantation personnelle et une vaste plantation communautaire placée sous la responsabilité du chef de village et protégée par un « interdit» (tapu). Le nom spécifique de ces plantations d'ignames (gaue'ufi, « travail de l'igname ») les distingue de tous les autres jardins, appelés gaue'aga ( « lieu de travail ») sans autre précision. Cette culture donnait le rythme à toutes les activités agricoles de l'année :
«L'importance des ignames, c'est une chose qui dirige le travail ! Si tu n'as pas d'ignames, tu ne vas pas travailler. Parce que tu vas travailler tes ignames, tu les apportes pour les garder à la maison, elles germent et tu vas préparer ton jardin ! Et si tu n'as pas d'ignames [...] tu restes à ne rien faire et c'est une saison [ou une récolte] pour rien ${ }^{22} . »$ (Pelesese Tokotu'u, extrait d'entretien)

La culture des ignames épuisant la terre, elle nécessite pour chaque récolte l'essartage d'une jachère « boisée » (akau' $i$, au moins trois ans de repos). Or, il y avait autrefois trois récoltes d'ignames annuelles préparées dans trois jardins différents :

- la «toute première récolte » ou " récolte qui vient en avant ", ta'u mu'amu'a (plantation en avril-juillet, récolte en novembre-décembre), mu'amu'a signifiant à la fois «ce qui vient en avant » et « ce qui est supérieur »;

- la « grande récolte » ta'u lahi (plantation de juin à août, récolte de février à avril) ;

- et la " dernière récolte » ta'u muli (plantation en septembre-octobre, récolte en avril-mai).

Tous les hommes préparaient au moins une récolte, généralement deux, trois s'ils s'en montraient capables, ce qui impliquait le défrichage d'une, deux ou trois parcelles chaque année. De nos jours, la plantation des ignames initie toujours le travail. Une fois les semences mises en place, la plantation est complétée par les plantations de kape, taros, bananiers, cannes à sucre, papayers et autres plantes, telles la courge et l'ananas, importées par les Occidentaux. Les kape et les taros, dont le développement est plus lent que celui des ignames (environ un an), sont laissés dans le champ quelques mois après la récolte des premières. Le jardin est ensuite abandonné. On intercale souvent entre la récolte et le repos du sol une plantation de manioc qui épuise peu la terre.

Les ignames demandent entre six et huit mois de croissance. Elles peuvent être conservées environ cinq mois une fois entreposées sous un « abri » (fa' ahi tahi ou fale'ufi: claie surélevée couverte d'un toit) - autrefois sous la charpente de la maison. Entre le quatrième et le sixième mois qui suivent la récolte, les semences sont préparées, les fruits d'une récolte étant, rappelons-le, utilisés pour préparer la récolte correspondante de l'année suivante. La préparation des semences distingue les têtes d'igname

21. Des engrais chimiques sont désormais employés par ceux des cultivateurs qui en ont les moyens financiers. De telles pratiques, toutefois marginales, complètent la rotation des jachères, mais ne s'y substituent pas.

22. «Ko te ma'uhiga o te 'ufi, ko te me'a taki gaue! E kapau mole iai hau 'ufi, mole ke alu gaue! [...] Hē e ke gaue au 'ufi, aumai tuku i fale, homo pea e ke alu o gaohi hau gaue! Pea kä mole hau 'ufi [...] nofonofo noa pe ko he ta'u noa. » (Pelesese Tokotu'u, extrait d'entretien). 
('ulu'i'ufi) premières germées, premières plantées et premières récoltées, des semenceaux issus des « corps » et appelés fakafale. Ces deux sortes de semences ne s'opposent pas seulement au sein de chaque récolte. Elles caractérisent aussi de façon radicale les différentes récoltes de l'année et l'ensemble du cycle agricole et rituel wallisien ${ }^{23}$.

L'organisation du travail est identique pour les trois récoltes: la plantation des têtes ('ulu'i'ufi) précède normalement celle des semenceaux (fakafale). La "toute première récolte » ta’u mu'aти'a (ти'a "aîné, précédant, antérieur »), est pourtant considérée comme une récolte de "têtes d'ignames " 'ulu'i'ufi, à la différence des récoltes suivantes ( $t a^{\prime} u$ lahi et ta'u muli), globalement considérées comme celles de fakafale. Comme le corps suit la tête, ces deux récoltes suivent la première récolte (ta'u mu'amu'a) qui initie tout le cycle. L'antériorité de la première récolte opère donc à deux niveaux. Elle combine antériorité chronologique (elle vient en premier) et prééminence spatiale, la " tête" 'ulu conjuguant élévation, projection avec ancienneté. Ces caractéristiques lui donnent, ainsi qu'à ses prémices, une importance toute spéciale.

P. Tokotu'u expliqua qu'autrefois, lorsque l'alimentation dépendait entièrement de la culture des jardins vivriers, la «toute première récolte » (ta'u mu'amu'a) était essentielle car, les têtes poussant plus vite que les autres semences, elle était la première arrivée à maturité : «C'est ta petite plantation pour manger » («Ko tau ki'i gaue kai ») ajouta-t-il, expliquant que si l'on se montrait paresseux ou que l'on se trompait en la préparant, on était voué à la famine entre les deux saisons de fruits à pain (mei). Si un homme ne planifiait qu'une récolte dans l'année, il fallait donc que ce soit celle-là, les deux autres récoltes étant secondaires si la première était suffisante. La première récolte apparaît ainsi comme celle qui règle toutes les autres activités agricoles et qui peut, éventuellement, toutes les remplacer.

Les deux autres récoltes, organisées de façon comparable, suivent et complètent la première de telle sorte qu'elles structurent le travail agricole sur le reste de l'année. La "grande récolte » tau lahi est particulièrement importante en ce que, disponible d'avril à octobre, elle fournit les ignames nécessaires aux prestations cérémonielles des nombreuses fêtes patronales et familiales de cette époque. Elle est plantée à la saison sèche, relativement fraîche et propice aux travaux difficiles. Cette récolte est celle $« \mathrm{du}$ moment où un homme est fort» («te temi malohi pe a te tagata»). La plantation de la « dernière récolte » ta'u muli a lieu en septembreoctobre. C'est « la récolte de la fatigue », de la lassitude («te ta'u ga'ega'e»). De ce fait, c'est aussi «la récolte des hommes grands travailleurs » ("te ta'u a te kau tagata fa'a gaue »), ceux qui se font remarquer par leur travail et leurs compétences. Selon sa réalisation, se différencient les hommes assurant le nécessaire et ceux que le courage au travail et l'orgueil poussent à un accomplissement dans ce domaine ${ }^{24}$. Cette dernière récolte était aussi autrefois la principale en quantité des trois récoltes communautaires. Bouclant la série des trois récoltes, elle est aussi appelée « la fin de la démangeaison de la terre » (te hiliga velikele). Avec elle commence le repos du dernier des trois jardins ouverts durant l'année.

S'appuyant sur le journal du père Chanel (1838-1839) et la correspondance d'autres missionnaires, Patrick V. Kirch (1994a) atteste l'ancienneté de ce type de calendrier sur la proche île de Futuna. L'oblation, début octobre, des fruits de la «toute première récolte " ta'u $m u^{\prime} a$ (plantation en avril-juin/premiers fruits en octobre ${ }^{25}$ ) constituait le moment majeur d'un cycle prolongé par les récoltes secondaires ta'u lasi (plantation en juillet-août/récolte en décembre) et ta'u muli (plantation en septembreoctobre/récolte en février). Kirch montre également l'articulation de ce calendrier avec le cycle rituel pré-chrétien. L'offrande des prémices d'ignames (ta'u mu'a) inaugurait, avec la saison des pluies, une saison rituelle intense (oblations, d'octobre à janvier ${ }^{26}$ ) suivie d'un relatif temps mort (fêtes de village de janvier à avril). Le retour des alizés (avril-mai) annonçait les plantations et

23. Bien que calendrier et pratiques diffèrent sous différents aspects, un parallèle peut être fait avec le cycle agricole et rituel kanak (Leblic, 2002 : 118-119). Mentionnons que les ignames destinées aux cérémonies des prémices sont plantées avant toutes les autres en Nouvelle-Calédonie et sont appelées " ignames vraies-ignames », dénomination proche de celle de leurs homologues wallisiennes, "vraies têtes d'ignames » (cf. infra).

24. Sans m'en préciser la raison, P. Tokotu'u indiqua que les ignames vakasoa et lautolu sont particulièrement cultivées pour cette récolte. Il ne m'a pas dit quelles espèces convenaient aux autres récoltes bien que la première doive comprendre les ignames qui, considérées comme originaires de Wallis, conviennent mieux que toutes autres aux prestations de début d'année (fai'ofa) : 'ufi poa, 'ufi laumahi, 'ufi voli, et encore 'ufi lausi, et kaumaile. On peut les accompagner de bananes hopa et d'ignames vegi.

25. Cette date correspond également à celle de l'oblation des prémices d'ignames (inasi) qu'à Tonga la population présentait autrefois au chef suprême (Douaire-Marsaudon, 1998).

26. Présentation de raies et de requins, puis les prémices des fruits de l'arbre à pain à Fakavelikele, la déité tutélaire du roi Niuliki ; distributions générales de nourriture, de kava et de porcs ; repas de féculents fermentés (masi) en l'honneur des déités responsables des cyclones; festivals de danse et subincision du pénis des garçons. 
divers préparatifs (fabrication de la poudre de curcuma et ramassage des œufs de tortue) avant la récolte.

Pour Wallis, nous ne disposons d'aucun témoignage permettant de reconstituer un tel calendrier agricole et rituel. Apparaissent, toutefois, des correspondances frappantes entre celui reconstitué par Kirch pour Futuna et celui documenté à Wallis en fin de $\mathrm{xx}^{\mathrm{e}}$ siècle. L'une d'elles est la similitude des récoltes (nombre, noms et rythme), bien qu'un décalage de deux mois existe entre la mise en terre de la récolte tau mu'a de Futuna et celle, ta'u mu'amu'a de Wallis. Une autre correspondance est la présentation collective des prémices de cette récolte (appelés " vraies têtes d'ignames "'ulu'i'ufi totonu, à Wallis). Dans les deux cas, enfin, ces oblations marquent un changement de cycle : à Futuna la présentation des prémices ouvrait autrefois la saison rituelle. À Wallis, elle marque de nos jours, sous la forme de prestations fai' $o f a$, les festivités chrétiennes de fin d'année et celles ouvrant la nouvelle année. Préparées et présentées à cette occasion, les prémices manifestent alors auprès du roi, du clergé catholique et de la chefferie la gratitude de la population pour les bienfaits prodigués et son attente d'une prospérité renouvelée. On retrouve donc pour l'oblation comme pour la plantation les deux mois de décalage entre le calendrier wallisien (décembrejanvier) et les anciennes cérémonies de Futuna (octobre).

Considérant l'importance de cette récolte, il est frappant que les informateurs wallisiens ne se soient pas accordés sur les dates de sa mise en terre : certains donnent avril-mai, d'autres, parfois les mêmes à un autre moment, donnent juinjuillet. La mise en parallèle des cycles wallisien et futunien permet une hypothèse : la « [toute] première récolte » ta'u [mu'a]mu'a devait autrefois être plantée, à Wallis comme à Futuna, en avrilmai (et récoltée entre octobre et décembre), conformément au cycle végétatif dans la région. Le plus probable est que les missionnaires catholiques, cherchant à substituer le calendrier chrétien à celui qui précédait, ont demandé à leurs néophytes de faire en sorte que l'offrande des prémices de cette récolte, importante parmi toutes, coïncide avec l'événement majeur de l'année chrétienne, la célébration de la naissance du Christ fêtée en Occident au solstice d'hiver, à quelques jours du changement d'année. Ainsi les dates données pour la plantation de la toute première récolte divergent-elles selon que l'on considère le calendrier ancien fondé sur le cycle végétatif (avril-mai) ou les pratiques actuelles adossées à la liturgie catholique (juin-juillet). Apparaît bien ainsi le lien ancien établi, dans la région, entre culture des ignames et temporalité cosmique et sociale. J'y reviendrai, après avoir développé les aspects montrant à quel point la culture et la circulation des ignames participent au travail rituel que la société met en œuvre pour constituer, renforcer et valider celles des relations sociales qui, marquées par l'ancienneté, fondent le bon ordre de la société et de son cosmos.

\section{Rites de propitiation et présentation des prémices}

La plantation des ignames initie le travail horticole de l'année. Ces tubercules - particulièrement les têtes issues de la première récolte précèdent toutes les autres récoltes dans le temps et les dépassent en valeur :

« C'est elles [les ignames] qu'Uvea [les Wallisiens] met en avant de tous les "féculents" [magisi]. C'est le premier "féculent" celui qui est le premier, les igna$\mathrm{mes}^{27}$. » (Aliki Liufau, extrait d'entretien)

La présentation des prémices d'ignames, qui initie toutes les récoltes de l'année, est essentielle. Cette prestation s'appelle polopolo, terme qui désigne les premiers fruits d'un jardin. On dit « $e$ au polopolo'i te gaue'aga» ce qui signifie « je polopolo la plantation » c'est-à-dire, «par cet usage spécifique des premiers fruits, j'ouvre la consommation de la récolte de ce jardin ». De nos jours encore, l'offrande de ces prémices ouvre rituellement la récolte car il s'agit du « premier travail pour déterrer sa "chair" [kakano] pour manger ${ }^{28} »$.

Toutes les prémices d'ignames (polopolo) sont des " têtes d'ignames » ('ulu'i'ufi). Toutefois, ici encore, les prémices de la première récolte diffèrent des suivantes en ce que, parmi les têtes d'ignames, elles sont les seules « vraies » (totonu) têtes d'ignames ('ulu'i'ufi totonu). Ces premiers fruits, pour chaque récolte, ne sont jamais consommés par les cultivateurs. Les prémices des deux dernières récoltes sont proposées aux visiteurs ou offertes dans l'entourage sur un mode informel. Il n'en va pas de même pour les prémices de la première récolte ('ulu'i'ufi totonu). Premiers entre tous, ces « fruits du travail de la terre » (te'u fua o te gaue kele, selon Aliki Liufau [extrait d'entretien]) constituaient avant la

27. «Pea ko ia e fakamu'amu'a ai e 'Uvea nei te magisi... [...] Ko te 'uluaki magisi aia e 'uluaki, te 'ufi. » (Aliki Liufau, extrait d'entretien).

28. «[...] ko tau uluaki gaue e utu mai ko tana kakano o kai [...]» (Pelesese Tokotu'u, extrait d'entretien). 
christianisation une prestation destinée aux déités 'atua en même temps qu'au " chef suprême " $(h a u)^{29}$. De nos jours encore, les fruits de la terre sont présentés de façon formelle lors des hommages et des vœux (fai'ofa) de nouvelle année adressés au roi, puis au clergé et aux « anciens » (chefs et représentants territoriaux). À travers eux, c'est à l'ensemble que forment la société et son cosmos dominé par Dieu que ces prestations s'adressent. Les modalités de celles-ci ont toutefois subi ces dernières années des modifications significatives.

Il y a quelques décennies, les premiers fruits ("vraies têtes d'ignames ») étaient prélevés sur la plantation communautaire avant d'être offerts au roi par chaque village à l'occasion de la nouvelle année, lors de la cérémonie dite fai'ofa. À défaut de récolte communautaire, seuls les chefs $\mathrm{du}$ district (chefs de village, chefs de district, membres du conseil du roi) se chargeaient de l'offrande au roi. De nos jours, il n'existe plus de jardins collectifs d'ignames. À la veille de la cérémonie, chaque homme s'efforce de fournir un panier de "vraies têtes d'ignames". Celles-ci sont regroupées, triées et réorganisées pour former des prestations convenables. Ce remplacement de l'offrande de prémices obtenues en commun par l'offrande d'une collection de prémices cultivées séparément et réunies pour l'occasion correspond aux transformations économiques et sociales à l'œuvre sur le territoire depuis une trentaine d'années. Si la récolte est suffisamment belle pour permettre une prestation correcte, chaque village la porte au roi et à l'évêque qui reçoivent ainsi des dons de toute l'île. Les autres destinataires (prêtres et religieuses, chefs de districts et membres du conseil royal) ne reçoivent d'offrandes que des résidents de leur district. Chaque village présente aussi une prestation à son chef et aux élus (conseillers territoriaux, député, sénateur...) résidant là.

Tous ces destinataires sont classés comme des « anciens » (mātu'a) représentant les niveaux imbriqués de la société : pays, districts/paroisses, villages. L'étude du cycle rituel (Chave-Dartoen, 2000) montre que le "pays », la "société » fenua, est compris comme une totalité dont Dieu règle le destin et dont le roi forme la clef de voûte. L'ensemble des anciens, et particulièrement les membres de la chefferie, sont conçus comme « travaillant » (gaue) à la prospérité et au bien de tous. Le terme gaue, qui signifie «travail», désigne avant toute chose le travail agricole des hommes. Ce terme toutefois n'est pas utilisé pour le roi. Tismas Heafala (1989: 44) précise qu'il serait à la fois irrespectueux et linguistiquement incorrect de lui appliquer le terme gaue. En effet, seul le mot ta'ata'a peut désigner son activité. Or, ce mot désigne également le "sang " dans le niveau de langue réservé à l'évocation du roi et $\mathrm{du}$ Christ - en référence à toute autre personne, "sang » se dit toto. Il apparaît ainsi qu'en ce qui concerne les récoltes annuelles, la responsabilité du roi diffère fondamentalement de celle des autres anciens, non en nature, mais en degré.

Pour assurer la prospérité des jardins et de leurs dépendants, les anciens «travaillent» (gaue), chacun à son niveau, en relation à Dieu. Ce travail, qui associe soin des jardins, rigueur morale et élévation spirituelle, appelle en retour la bénédiction divine (manu'ia) sur chaque ancien et ses dépendants. Les prémices offertes à un ancien soulignent, avec sa position et ses responsabilités, les qualités personnelles qui attirent sur lui une « faveur divine » (manui'a) dont il fait bénéficier tous ses dépendants. Ce faisant, l'oblation des prémices exprime la reconnaissance et la gratitude (fakamalo) de la population pour les bienfaits passés, consolidant la renommée et le statut personnel qui, pour chaque ancien, viennent conforter son principe " âme » laumālie. Cependant, ces prestations sollicitent surtout, avec la mansuétude (fakalelei) de chaque destinataire, son «intercession» (hufaki mai) auprès de Dieu. Dieu reste donc, comme autrefois les déités, le destinataire ultime de prestations présentées dans un but propitiatoire.

Le roi, en position d'ancien absolu, constitue au sommet de l'organisation sociale la référence sur laquelle se règle, avec l'enchâssement des responsabilités, la gradation du rang et des statuts. Il assure la fertilité selon le même principe, mais à un autre niveau de responsabilités

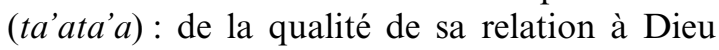
dépendent son gouvernement éclairé, la prévention des désordres et des conflits, l'absence d'épidémies, de sécheresses et de cyclones. Cette médiation bénéfique porte le nom spécifique de tapu'akina. C'est donc en premier lieu au roi, qui garantit les conditions sociocosmiques de la prospérité générale, que sont destinées les vraies têtes d'ignames de la toute première récolte.

Les récoltes d'ignames sont ainsi considérées comme une manifestation des relations bénéfiques entre le pays - aux premiers rangs duquel les horticulteurs et les anciens - le roi et Dieu. L'ordre donné à la présentation des prémices exprime, par cette reconnaissance générale et graduée, les différents niveaux de responsabilité

29. Voir également Joseph Henquel (ms, circa 1910). Ces faits confortent la comparaison avec le calendrier rituel futunien reconstitué par Kirch ( $c f$. supra). 
et l'articulation des relations qui les organisent. Les prestations fournies pour le fai'ofa soulignent la réciprocité, sans cesse affirmée et renouvelée, des relations qui font vivre et prospérer la société, le « pays » fenua : réciprocité interne à la société suivant les responsabilités respectives et emboîtés des anciens ; réciprocité entre la société que forment tous ensemble population des fidèles, anciens, roi à leur tête ${ }^{30}$ et Dieu.

Initiant et clôturant les activités agricoles de l'année, la «toute première récolte» $(t a$ 'u mu'amu'a) est essentielle dans l'organisation du cycle des cultures. Son importance réside toutefois essentiellement dans le caractère particulier de ses semences, faites de " têtes d'ignames ». En naîtront les ignames qui, destinées aux anciens de la communauté et à Dieu, manifesteront l'achèvement et la puissance de ces derniers pour en renouveler les bienfaits. La temporalité de cette première récolte fait coïncider calendrier rituel, cycle de la végétation et cycle de la vie sociale. La présentation formelle des ignames, en particulier lors des prestations de prémices, manifeste alors les aspirations de la société qui, face à Dieu, régule, manifeste et conforte son ordre interne et la gradation des statuts personnels, soulignant dans sa cyclicité annuelle, générationnelle et dans son renouvellement permanent, l'importance de l'ancienneté relative qui règle l'alternance de la vie et de la mort, du déclin et du renouveau tout en assurant la pérennité de la société et de son organisation.

\section{Hommes et ignames: un même système de relations}

À la différence des Occidentaux, les Wallisiens ne se définissent pas comme des êtres faits de substances, mais comme des êtres constitués de relations (Chave-Dartoen 2000). Ces relations, de différentes sortes, établissent le statut, les diverses formes d'attachement, l'apparence et le souffle même de la vie ${ }^{31}$. En partie transmises par les ancêtres et les parents, elles sont sans cesse redéfinies dans les interactions avec le monde social et les entités du cosmos qui prési- dent à la destinée, particulièrement lors des cérémonies et des passages du cycle de vie. La constitution des hommes et des femmes relève donc de considérations sociologiques, non pas biologiques : ils s'insèrent, tout au long de leur vie et au-delà de la mort, dans un réseau dense de relations qui, seul, les définit et leur donne une existence. J'ai pu mettre en évidence que les rites de passage prennent en charge trois principes différents et complémentaires : le « corps » sino, le «sang» toto et l'« âme» laumālie (ChaveDartoen, 2000). Les deux premiers correspondent à des composantes physiquement manifestes, mais renvoient simultanément à des relations constitutives sans lesquelles la personne ne saurait trouver de place dans la société. Le principe « corps » correspond à la forme sensible que prennent les différentes entités du monde. Pour les vivants, le corps est conformé de l'intérieur par l'alimentation paternelle, de l'extérieur par le vêtement et les cosmétiques procurés par la mère $^{32}$. Le principe «sang " s'élabore et se perpétue en relation aux terres héritées des ancêtres, à la terre natale en particulier. Ces relations passent par la mère et le côté maternel de la parenté. Le principe « âme » (laumālie) existait avant que la foi catholique ne lui donne une signification particulière. Il désigne une relation étendue (lau) et continue (mâlie) à des ancêtres dont les plus prestigieux étaient autrefois déifiés et auxquels se raccrochaient les descendants, particulièrement les aînés masculins qui leur succédaient en position d'ancien dans la tâche de diriger la communauté. Ce principe, en s'appuyant sur les relations généalogiques les plus prestigieuses, renvoyait également à des relations étendues dans l'espace. Dépendants et alliés dispersés dans l'île, reconnaissant la position et le statut des anciens ${ }^{33}$, établissaient leur renommée. Or, statut social (hérité des paternels) et principe « âme » étaient autrefois liés. Inexistante ou insignifiante pour les "gens du commun» $\left(t u^{\prime} a\right)$, l'« âme » laumālie distinguait, par les relations valorisées qui la constituaient, les membres de l'ordre social supérieur ('aliki): ces relations, constitutives du principe " âme », ordonnaient les hommes en unegradation de statut et de rang

30. Mis à part quelques cas de conversions récentes ou de retour d'expatriés convertis outre-mer (Témoins de Jéhovah, Viens et vois...), la plupart des insulaires sont catholiques romains pratiquants.

31. Une comparaison intéressante peut être faite, à ce sujet, entre les conceptions occidentales, wallisiennes et abelam (région de Maprik en Papouasie Nouvelle-Guinée) de la constitution des personnes. Dans ce dernier cas (Coupaye, 2009), une composante de la personne, très directement impliquée dans la croissance des grandes ignames cérémonielles, est clairement substantielle (le jëwaai, alternativement sang, odeur et chair), tandis qu'une autre, telles le yakët, est une qualité du corps reposant sur des principes plus proprement relationnels.

32. La sépulture constitue un corps pour les morts, le « reposoir » vakafaka'aga, un pour les anciennes déités.

33. Faute de ces marques de reconnaissance, le statut des anciens déclinait au profit de ceux capables de renforcer, avec leur propre renom, celui de leurs descendants. Les usurpations par destitution ou assassinat sont fréquentes dans les traditions orales. 
suivant l'aptitude qu'ils montraient à succéder à leurs aînés dans la direction et la représentation de leurs dépendants.

L'étude des prémices fai'ofa montre que les différences de statut et de rang, quoique aujourd'hui atténuées, restent importantes et distinguent entre eux les hommes en charge de responsabilités. Ces distinctions basées sur les critères d'ascendance (principalement d'ascendance paternelle), de relation aux ancêtres, de capacité personnelle s'appuient sur l'aînesse et l'ancienneté relative (j'y reviendrai). Elles correspondent toujours à un principe «âme» laumālie, mais christianisé en ce que la relation à Dieu prime désormais sur tout autre aspect. Si les références ancestrales fondent toujours la gradation des statuts, l'âme trouve de nos jours, pour chaque fidèle (homme et femme), son origine et sa référence ultime en Dieu. Je voudrais maintenant montrer comment s'établit le lien étroit qui, associant la circulation des ignames à ce principe "âme ", distingue les hommes des femmes et différencie les hommes entre eux.

\section{La tête en avant : antériorité des ignames et des hommes}

L'opposition entre les semences 'ulu'i 'ufi et fakafale forme un contraste qui rejoint, sous certains aspects, l'opposition dans les principes constitutifs qui définissent hommes et femmes, non pas comme des catégories biologiques exclusives et absolues, mais comme les éléments complémentaires d'une unité sociologique, le couple. Le couple est socialement défini dans sa relation à la maisonnée qu'il occupe, à sa descendance commune, et aux obligations cérémonielles que, de ce fait, il partage ${ }^{34}$.

La tête est généralement conçue comme l'«avant» $m u^{\prime} a$ et le " haut " 'ake de la personne. Elle est en relation avec l'" âme » laumālie. Constituée d'un contenant osseux et sec, elle s'oppose au reste du corps qui apparaît comme un contenu (" masse de chair sanglante », toto kakano) soutenu par une charpente osseuse. Tandis que la " tête »'ulu s'ouvre sur l'extérieur et présente par ses orifices ce que l'être offre au monde, le corps est fermé sur un «intérieur» (loto) comprenant les viscères, les organes de la reproduction et surtout le "siège de la vie" (ma'uli'aga). C'est là, dans le ventre féminin, que le fotus prend vie et se développe à partir du sang mêlé du père et de la mère. L' " intérieur » (loto) d'une femme, épouse et mère, reste dans une relation étroite avec la « maison » (fale) et la terre natales qu'autrefois elle quittait fort peu ${ }^{35}$. Cette relation passe par le sang (menstrues, défloration, couches, inhumation) qui va rejoindre dans la terre celui des ancêtres. Ainsi, pour une femme, les relations « de sang » établies dans la maison priment au regard de ce qui se passe pour son conjoint qui, lui, est sans relation directe avec la terre de résidence.

La "maison » fale, conçue comme fraîche et humide, fait partie de la terre dans laquelle on l'installe (on l'« enterre » tanu) et les semenceaux fakafale lui sont associés du fait de leur lente germination sous la toiture. Les ignames qui en résultent (fakafale) et dont le corps se développe dans la maison avant de rejoindre la terre, sont comparables au fotus, en relation (« de sang ») avec le double réceptacle de l'utérus et de la terre maternelle. Ce parallèle entre le corps des ignames et les enfants nés des femmes de la maison paraîtra mieux fondé encore si l'on ouvre maintenant la comparaison aux têtes d'ignames. Pour ces dernières, en effet, les relations déterminantes diffèrent nettement.

Si le corps, charnu, humide et sanglant ${ }^{36}$ correspond à des relations maternelles, la tête et la tige lianescente de la plante correspondent à la conception locale des relations masculines et paternelles. En contraste avec le corps du tubercule, s'enfonçant dans la terre ameublie en quête d'humidité, la liane, issue d'une tête gardée au sec (les 'ulu'i'ufi sont mises à germer dans du sable, et plantées sous un petit tas de sable qui draine l'humidité) se développe vers le haut, la lumière et la chaleur. À ce développement vers le haut, soutenu par des tuteurs et opéré par étapes successives font pendant, pour les hommes, la nécessité de références généalogiques prestigieuses et la succession d'ancêtres paternels vers lesquels on remonte pour déterminer le statut social. Ainsi, le développement des ignames, et particulièrement de celles issues de têtes ('ulu'i'ufi), paraît correspondre à celui, pour les

34. Les sociétés de Polynésie occidentale forment un cas d'école dans le domaine de l'étude du genre (« sexe socialement défini »). L'opposition de sexe y prend en effet deux formes exclusives : l'une, homme/femme correspond à notre opposition masculin/féminin sans toutefois reposer sur les fonctions biologiques (la reproduction n'est pas comprise comme résultant nécessairement d'une union sexuelle entre mâle et femelle) ; l'autre, entre collatéraux de sexe opposé, est pensée de façon radicalement différente (Chave-Dartoen, 2000).

35. Le mode de vie change beaucoup depuis une trentaine d'années (démographie, règles de résidence, salariat féminin...), les femmes devenant plus mobiles.

36. De nombreuses variétés d'igname ont une chair dite « rouge » kula, couleur du sang. Ce type de parallélisme peut être poussé sur d'autres critères, tels le parfum et la peau. 
hommes, des relations aux ancêtres prestigieux. La correspondance entre les " têtes d'ignames » 'ulu'i'ufi et le côté paternel de la parenté ne s'arrête toutefois pas au déploiement aérien de la tige.

Chargé de relayer son père à la tête du groupe qu'il « dirige et représente » (pule), tout homme est considéré, à un certain degré, comme un ancien. Or, à Wallis, les anciens sont également des « têtes », comme le formulent explicitement les locutions 'ulu'i mātu'a (" têtes des anciens » dirigeant la branche généalogique) et 'uluaki (" premier-né », " celui qui est à la tête, qui dirige sa fratrie »). À l'exercice de l'autorité (pule), correspond l'idée d'antériorité temporelle et spatiale rendue par le verbe taki, « diriger », la locution taki mu'a signifiant à la fois "marcher en tête, aller en éclaireur » et " excellent, supérieur, prédominant » (Rensch, 1984). Les ignames les plus valorisées, celles qui, issues des têtes ('ulu'i'ufi), donnent la première récolte, vont précisément à ceux des anciens qui vont en tête, aux supérieurs (taki mu'a), le roi et l'évêque en premier lieu. Il faut souligner à nouveau que l'antériorité temporelle et spatiale définit à la fois les anciens dotés de responsabilités et les prémices de la récolte.

Un autre point significatif est le renouvellement nécessaire des semences d'ignames tous les trois ans pour que les récoltes ne dépérissent pas. Cet intervalle de trois générations rappelle celui qui, chez les humains, se ferme et se réinitialise lorsque l'on transmet à l'aîné des petits-enfants le nom du grand-père ou de la grand-mère paternel(le) (un « ancêtre » kui). Cette alternance des générations dans les noms ancestraux perpétue l'existence sociale des ancêtres et leur influence auprès de leurs descendants tout autant qu'elle confère aux descendants ainsi distingués, particulièrement aux hommes, le statut et l'autorité des ancêtres les ayant précédés dans le nom. Pour les ignames comme pour les hommes, la succession de trois générations apparaît donc comme nécessaire à la relance d'un cycle qui se boucle préférentiellement entre l'aîné d'une fratrie et l'ancêtre paternel valorisé dont il hérite du nom et de l'autorité (Chave-Dartoen, à paraître).

L'analyse peut être poussée si, considérant les trois « récoltes " $t a$ ' $u$ de l'année comme à la fois comparables (car de " même génération ») et différentes (car issues de jardins différents), on les rapporte à l'ordre des enfants dans une fratrie. Une alternance règle l'attribution des noms aux enfants d'un couple : les paternels donnent un nom à l'aîné, les maternels au puîné, l'alternance s'étendant aux cadets. L'antériorité ( $m u^{\prime} a$ ) est soulignée pour l'« aîné de la fratrie » (te mu'a, te 'uluaki) comme pour la «toute première récolte » (ta'u mu'amu'a). Ils sont, de ce fait, supérieurs en statut, car en relation étroite avec les anciens de la société, les ancêtres et Dieu qui en règlent le destin. Le « deuxième [enfant]» (te lua) et la deuxième récolte (ta'u lahi) sont de statut inférieur bien qu'ils soient valorisés, l'un pour la relation qu'il manifeste entre les deux côtés de la parenté dont il renforce les liens en affirmant la complémentarité et la solidarité cérémonielle (autrefois militaire), l'autre pour sa profusion et la participation significative qu'elle rend possible lors des échanges cérémoniels impliquant ces deux mêmes côtés. Enfin, les «autres [enfants]» (ae muli) et la troisième récolte (ta'u muli), bien qu'inférieurs aux précédents, assurent et manifestent la prospérité d'une maisonnée par les mariages et les naissances qu'ils promettent. Dans les deux cas ils sont, pour une maisonnée, la garantie de sa pérennité dans la relance des échanges cérémoniels dont elle est l'origine et le centre.

L'organisation des générations est donc comparable pour les ignames et pour les humains. L'aînesse en est le premier aspect. Il distingue, à chaque génération, les premiers-nés des hommes et des ignames comme des anciens en relation aux ancêtres qui, situés deux générations en amont, sont des anciens par excellence : "tête des anciens " 'ulu'i mātu'a paternel pour les humains; "vraie tête d'igname" 'ulu'i'ufi totonu, pour les ignames. Au principe de l'aînesse vient s'ajouter la complémentarité de cotés cérémoniels opposés. Elle fait correspondre position du puîné et deuxième récolte: tandis que le puîné « revient » $m a^{\prime} u$ au côté maternel de sa parenté (il s'oppose à son aîné en complétant les relations nécessaires à l'assise de son autorité), la " grande récolte » (ta'u lahi) assure la mise en circulation de prestations où les deux côtés de la parenté sont à la fois rivaux et solidaires. Enfin, le renouvellement et la prospérité de la société sont assurés par « la suite » (te muli) des enfants comme des ignames qui, circulant en grand nombre à partir des maisonnées prospères, renforcent le statut du groupe social et les relations fondamentales de la société que l'aînesse et la complémentarité cérémonielle mettent en place à différents niveaux.

\section{Les ignames, fils des hommes}

Il convient enfin de préciser la relation, esquissée plus haut, entre les hommes et des récoltes issues d'un travail génésique particulier afin d'en 
pousser l'analyse. Lorsque les hommes usent de leur pieu pour planter les ignames, ils accomplissent un acte proche de l'acte sexuel (gaue'ifo). Ouvrant la terre, ils déposent une semence faite de chair et d'un petit germe comme, ouvrant un passage dans le ventre de leur partenaire (on dit d'une vierge qu'elle "n'est pas encore ouverte " heki avahi), ils y déposent, avec le sperme, une promesse de principe « âme » et du « sang » toto - il ne s'agit alors pas de cellules sanguines, mais d'une relation de filiation du type appelé «sang» (toto). Le tubercule se développe ensuite dans la terre comme le bébé se développe dans le ventre de sa mère. Enterré peu profondément et recouvert d'un peu de sable, il est aussi comparable aux vivants « enterrés » (tanu) dans leur « maisons » fale et aux " morts » mate inhumés dans leurs sépultures. En vieillissant, la plante prend du «corps » sino sous terre et s'élève en surface le long des tuteurs. Après une gestation de plusieurs mois au cours desquels la terre prend en charge la croissance de la plante, tandis que les hommes s'appliquent à dégager les tiges des mauvaises herbes et à les fixer sur les tuteurs, la maturité est atteinte. La partie visible de la plante forme alors une colonne de lianes intriquées vaguement anthropomorphe en ce que, dressée sur plusieurs mètres, elle se termine par une masse suggérant une tête. L'offrande des prémices assure la levée de l'interdit (puhi, « trouer, percer») permettant la récolte. Cette dernière est glosée comme une "ouverture» (avahi) qui renvoie à l'ouverture permettant la naissance du bébé. Les prémices (des «têtes" 'ulu) sortent de terre comme la tête du nourrisson en train de naître.

Rappelons que, pour la récolte, chaque tubercule est tiré de terre avec précautions. Le sabre d'abattis, glissé le long de la racine, détache les radicelles comme on coupe le cordon ombilical pour séparer définitivement le nourrisson de la parturiente. Le tubercule est alors doté, comme le bébé, d'une peau fine et blanche (kili mulamula), d'un corps et d'une tête. Les tiges qui s'en sont progressivement élevées pour s'épanouir hors de terre ont séché et disparu. Si l'on pousse le parallèle entre le développement de la personne humaine et celui des ignames, le tubercule sortant de terre pour entrer dans la société des hommes paraît toutefois gagner une forme de complétude qui exclut tout principe d'origine maternelle. Il ressort alors d'un niveau d'existence supérieur, très masculin, conditionné par ses qualités propres (taille, peau, parfum...), son ascendance ('ulu'i'ufi, fakafale, type de récolte) et sa circulation au sein de la société. Désormais le critère de l'antériorité relative distingue les

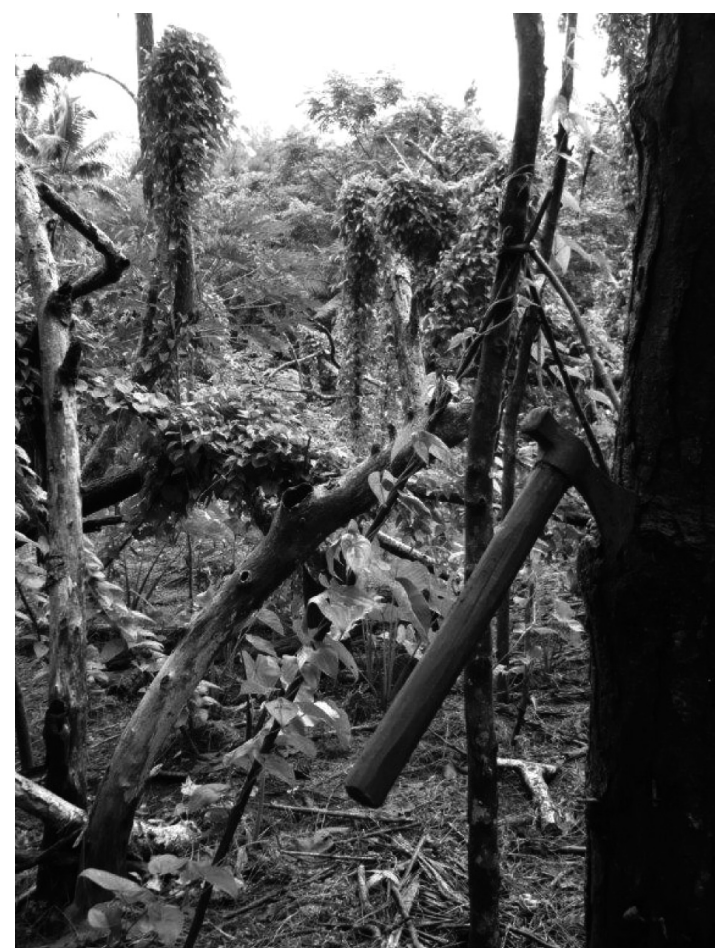

Рното 3. - Les plants d'ignames forment, avant que leur végétation ne dégénère, de hautes silhouettes anthropomorphes dont la tête se dresse au dessus des autres cultures (cliché de l'auteur, janvier 2008)

ignames entre elles comme il détermine les anciens à destination de qui elles sont réservées, définissant ce qui s'apparente pour l'igname à un principe « âme » comparable à celui des hommes qui les cultivent et les font circuler. Associant dans une même logique contraintes biologiques et considérations sociologiques, on voit ici comment la valorisation de l'antériorité ignore le déterminisme de la nature : ici, le cycle végétatif des ignames et le bon ordre du monde sont des principes proprement sociaux, la société formant la totalité englobante à partir de laquelle l'ordre du cosmos peut être lu.

À la différence des enfants humains, les ignames n'ont toutefois pas de relations de type " sang », essentiellement conférées par la mère et les ancêtres maternels. Leur font également défaut le souffle qui assure aux nourrissons la pulsation de la «vie » ma'uli. Les ignames, et particulièrement les "têtes d'ignames", sont ainsi comme des enfants, mais des enfants sans mère et sans souffle. Le vocabulaire en témoigne : les tubercules convenables pour une prestation (principalement les ignames) doivent être " développés, gros, volumineux» foha. Or ce mot désigne également un descendant masculin en $\mathrm{G}+1$ pour Ego masculin. En cultivant les ignames, les hommes conforment ainsi dans la 


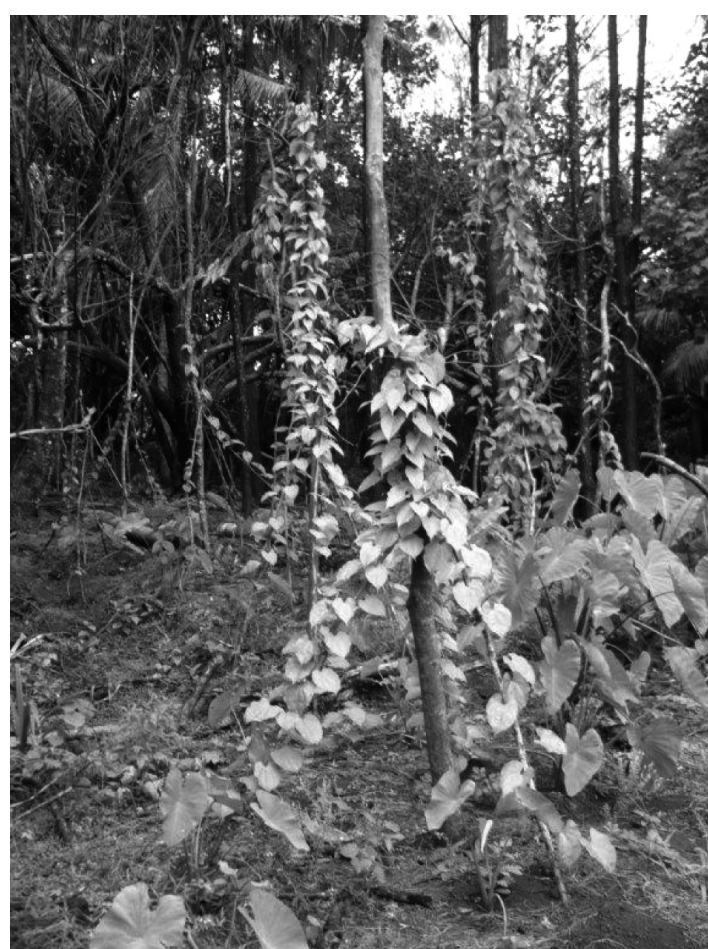

Рното 4. - Plants d'ignames en cours de croissance. Les tuteurs sont disposés en faisceaux au pied desquels les tiges s'élèvent du sol soigneusement ameubli (cliché de l'auteur, janvier 2008)

terre les « biens de valeur » (koloa) qui, constitutifs de leurs prestations cérémonielles, sont à certains égards pour eux comparables, par les relations ainsi établies et transmises, à leurs propres enfants masculins foha. Parmi ces tubercules, cependant, les têtes d'ignames se distinguent particulièrement par la supériorité de leur principe " âme » face à la dominance du principe «corps» des ignames fakafale et des autres tubercules (aracées). Proches des anciens, elles sont, sous cet aspect, équivalentes à des fils aînés. Cette différence explique la dévalorisation relative et la place secondaire des autres ignames, des taros et des kape dans le circuit des échanges cérémoniels.

Cette analyse montre que les caractéristiques distinctives des ignames obéissent à des principes qui intéressent essentiellement les hommes. Hommes et ignames sont comparables par les relations étendues (relations multiples à des ancêtres valorisés et au "pays » fenua) qui les définissent, les constituent et que leur circulation instaure. Comme les hommes aussi, les ignames, ordonnées en statut selon l'ordre de naissance et l'ascendance, circulent entre des unités sociales. Ainsi, passant d'une terre à l'autre, les semences d'ignames circulent, comme les hommes partent s'installer sur les terres de leurs compagnes pour y développer une double descendance : les garçons, d'une part, se dispersent au gré des mariages, les ignames, de l'autre (tubercules et semences) se disséminent au gré des échanges.

\section{Conclusion}

L'analyse entreprise ici ne distingue pas aspects imaginaires et ceux, proprement matériels et techniques, de l'horticulture à Wallis. Bien plus, elle montre que dans ce cas précis, matériel et technique ne peuvent être pleinement saisis et compris sans la référence permanente aux représentations collectives et aux relations sociales qui leur donnent sens et en organisent, partiellement au moins, l'agencement. Les hommes de Wallis obtiennent en effet dans leurs jardins, non pas des produits de consommation dont la valeur réside dans la qualité ou la quantité, mais des « biens de valeur » (koloa) dont la circulation contribue au renouvellement de la société et qui sont, à ce titre, pleinement intégrés au monde social ${ }^{37}$. Les principes constitutifs de ces biens de valeur correspondent à ceux socialement essentiels pour des hommes qui les cultivent. Par les relations ainsi établies, les tubercules destinés à se disperser - surtout les "têtes d'ignames" - sont, pour les hommes, comparables à des « fils » $(f o h a)$ sans mère. Obtenus directement de la terre, ils circulent, vivants (jamais cuits), dans les cérémonies propitiatoires au premier rang desquelles figurent les remerciements de fin d'année fai'ofa. Cultivés dans les jardins lointains et constamment renouvelés, formant des prestations collectives valorisées, ils relèvent moins de la terre du jardin que de la totalité du " pays » fenua. Ils forment ainsi les prestations dont le pays, la société fenua, fait l'offrande en remerciement des bienfaits que la chefferie et les représentant territoriaux - à un niveau local-, le roi hau à un niveau supérieur et englobant, procurent à la population avec la bénédiction de Dieu.

37. Pour une approche différente mais convergente de cette question, voir le remarquable travail de Ludovic Coupaye (2009a et b) sur la culture des ignames chez les Abelam de Papouasie Nouvelle-Guinée. Son approche associe analyse technique et étude des représentations, notamment des logiques locales de l'action. Elle dégage, entre autres, le "potentiel sociogénique » (sociogenic potential, 2009a : 96) que les investissements techniques, cognitifs et sociaux (relationnels), confèrent aux artefacts en les amenant à l'existence sociale. 


\section{BIBLIOGRAPHIE}

BARRAU Jacques, 1963. L'agriculture des îles Wallis et Futuna, Journal de la Société des Océanistes 19, pp. 157-171.

Chave-Dartoen Sophie, 2000. Uvea (Wallis), une société de Polynésie occidentale, étude et comparaison, mémoire de doctorat en Ethnologie et Anthropologie sociale Paris, EHEss.

—, à paraitre. La pluralité référentielle des noms propres. Constitution, statut et pérennité de la personne à Wallis, in D. Monnerie, C. Leguy et S. Chave-Dartoen (éds), Nomination et organisation sociale.

COUPAYE Ludovic, 2009a. What's the matter with technology? Long (and short) yams, materialisation and technology in Nyamikum village, Maprik district, Papua New Guinea, The Australian Journal of Anthropology 20, pp. 93-111.

—, 2009b. Ways of enchanting. Chaines opératoires and Yam Cultivation in Nyamikum Village, Maprik, Papua New Guinea. Journal of Material Culture 14 (4), pp. 433-458.

Douaire-Marsaudon Françoise, 1998. Les premiers fruits : parenté, identité sexuelle et pouvoirs en Polynésie occidentale (Tonga, Wallis, Futuna), Paris, CNRs Éditions-Éditions de la maison des sciences de l'homme.

Guiot Hélène, 1998. Forêt taboue et représentations de l'environnement à 'Uvea (Wallis), Journal de la Société des Océanistes 107, pp. 279-298.

GuIot Hélène et Isabelle LeBLIC (éds), 2006. Dossier Wallis-et-Futuna, Journal de la Société des Océanistes 122-123, pp. 1-186.

GrIJP Paul (van der), 2002. Selling is Poverty, Buying a Shame: Representations of Work, Effective Leadership and Market Failures on Wallis, Oceania 73, 1, pp. 17-34.
Heafala Tismas, 1989. Rencontre de deux cultures : le catéchisme de Monseigneur Bataillon, mémoire de maîtrise, Paris, Institut catholique.

Henquel Joseph, circa 1910. Talanoa ki Uvea nei, manuscrit dactylographié, mission de Lano, Wallis.

Juillerat Bernard, 1986. Les enfants du sang. Société, reproduction et imaginaire en Nouvelle-Guinée, Paris, Éditions de la maison des sciences de l'homme.

—, 2001. Penser l'imaginaire. Essais d'anthropologie psychanalytique, Lausanne, Éditions PayotLausanne.

KIRCH Patrick Vinton, 1978. Indigenous agriculture on Uvea (Western Polynesia), Economic Botany 32, 2, pp. 157-181.

—, 1994a. The Pre-christian Ritual cycle of Futuna, Western Polynesia, Journal of the Polynesian Society 103, 3, pp. 255-298.

-, 1994b. The Wet and the Dry, Irrigation and Agricultural Intensification in Polynesia, Chicago and London, The University of Chicago Press.

LEBLIC Isabelle, 2002. Ignames, interdits et ancêtres en Nouvelle-Calédonie, Journal de la Société des Océanistes 114-115, pp. 115-127.

Lевот Vincent, 2002. La domestication des plantes en Océanie et les contraintes de la voie asexuée, Journal de la Société des Océanistes 114-115, pp. 46-61.

ReNsCH Karl, 1984. Tikisionalio fakauvea-fakafalani, Dictionnaire wallisien-français, Canberra, The Australian National University, Pacific Linguistics Series, Special number. 